\title{
Characterization of Thin Films for Solar Cells and Photodetectors and Possibilities for Improvement of Solar Cells Characteristics
}

\author{
Aleksandra Vasic ${ }^{1}$, Milos Vujisic ${ }^{2}$, \\ Koviljka Stankovic ${ }^{2}$ and Predrag Osmokrovic ${ }^{2}$ \\ ${ }^{1}$ Faculty of Mechanical Engineering, University of Belgrade \\ ${ }^{2}$ Faculty of Electrical Engineering, University of Belgrade
}

Serbia

\section{Introduction}

Faced with an alarming increase of energy consumption on one side, and very limiting amounts of available conventional energy sources on the other, scientists have turned to the most promising, renewable energy sources. Possibilities for the application of solar systems based on photovoltaic conversion of solar energy are very wide, primarily because of their relatively low cost and very important fact that solar energy is most acceptable source of electrical energy from the environmental point of view. Recently, increased investments in the development of PV technology are observed worldwide. Photovoltaic (PV) conversion of solar energy is one of the most up-to-date semiconductor technologies that enables application of PV systems for various purposes. The wider substitution of conventional energies by solar energy lies in the rate of developing solar cell technology. Silicon is still the mostly used element for solar cell production, so efforts are directed to the improvement of physical properties of silicon structures. Silicon solar cells belong to a wide group of semiconductor detector devises, though somewhat specific in its design (larger than most of the detectors). Basic part of solar cell is $\mathrm{p}-\mathrm{n}$ junction, which active part is less that $0.2 \mu \mathrm{m}$ thick, so it could be treated as thin film. This photosensitive layer have the most important influence on solar cell functioning, primarily on creation of electron-hole pairs under solar irradiation, transport properties in cells, formation of internal field, and finally, output characteristics of the device such as short circuit current, open circuit voltage and efficiency. Furthermore, in order to function as a voltage generator with the best possible performances, beside p-n junction other thin films such as contact, antireflective, protective (oxide) thin films must be applied both on the front and on the back surface of solar cells. Also, in order to improve characteristics of the device, MIS structure (thin oxide layers) and back surface field layers are routinely used.

Since thin films are very important in many fields of modern science (solar cell technology, for example), a large number of methods were developed for their characterization. Characterization of thin films includes investigations of physical processes in them, developing of the methods for measuring major physical and electrical properties and their 
experimental determination. From the aspect of quality assessment of semiconductor device performance, characterization of the whole device gives best results especially in working conditions.

\section{Characterization of thin films for solar cells and photodetectors}

Contemporary trends in microelectronics and electronics in general are oriented to thin films, both from technological and scientific standpoints. Thin film devices as a whole or just a parts of the of devices such as surface, protective, antireflective, contact, or other thin films, have significant advantages over bulk materials. Beside obvious advantage in material and minimization of the device dimensions, methods for obtaining thin films are simpler and less demanded when the quality of the material is concerned than for thicker films. Moreover, characteristics of thin films could be significantly different from the bulk material and could lead to better performances of the device.

Great importance of thin films in modern science, as well as diversity of their characteristics made necessary the development of numerous methods for their research. Investigations of both physical and electrical properties of thin films are necessary primarily in order to determine the best combination for given working conditions (for example, high temperature, exposure to radiation, etc.). On the other hand, ion implantation, laser beams, epitaxial growth in highly controlled environment, etc., are commonly used for structural changes and obtaining better output characteristics of the devices. All of that was made possible by development, availability and improvement of sensitivity of methods for composition and structural characterization of materials. Although also very important in the process of thin film formation, these methods are essential for the quality assessment of the whole device in working conditions. In solar cells, for example, measurement of the output characteristics such as ideality factor, serial and parallel resistance, fill factor and efficiency, could directly or indirectly indicate the possibilities for the improvement of the production technology (from the basic material, formation of thin films, contact films, etc.). The choice of the appropriate method in each case depends on the type of the investigations and expected results. The most commonly used method for characterization of electrical properties of semiconducting devices (such as solar cells) is current-voltage $(I-V)$ measurement. Versatility of the data obtained in this way gives very important information about the device (solar cell), both from the fundamental standpoint (ideality factor, series and parallel resistance) and from the standpoint of the output characteristics (short-circuit current, open-circuit voltage, fill factor, efficiency).

Also, since contact films have significant influence on the output characteristics of all semiconducting devices, they must possess certain properties such as: low resistivity, good connection to the basic material, temperature stability, and low noise. One of the most important characteristic of detectors such as solar cells is their energy resolution that primarily depends on noise. That is why measuring and lowering noise is important for obtaining good quality detectors. It is known that low frequency noise ( $1 / \mathrm{f}$ and burst noise) is manifested as random fluctuation of the output current or voltage, leading to lowering of the efficiency of the device. Because of the large surface to volume ration, surface effects are expected to be a major cause of $1 / \mathrm{f}$ noise, so good quality contacts are of great importance. That is why measurements of $1 / \mathrm{f}$ noise and improvement of silicides characteristics by lowering $1 / \mathrm{f}$ noise in them leads to the production of reliable contacts. 


\subsection{Noise in thin film semiconducting devices}

Negative influence of noise on the photodetector characteristics could be observed in widening of the spectral line of the signal as well as in rising of the detection threshold. That is the reason why investigation of physical basis of different types of noises is necessary for their minimizing. Noise level primarily depends on fabrication procedures and is connected to the fundamental physical processes in semiconducting devices, so it could be said that noise appears in every detector regardless to their type or quality. Noise is commonly classified into three categories: thermal noise, frequency dependent noise and shot noise. Also, noise could be classified according to the physical processes as generationrecombination noise, diffusion noise and modulation noise. Low frequency noise, $1 / \mathrm{f}$ and burst noise are especially important in semiconducting devices. Various experiments suggests (Jayaweera et al., 2005, 2007) that the origin of this noise is fluctuation of the number free charge carriers connected to existence of the traps located in the vicinity or directly in the junction area, or fluctuation of the mobility of charge carriers. In both cases these fluctuations arise from the interactions of carriers with defects, surface states and impurities, that are either introduced during manufacturing of the device, or as a consequence of the hostile working conditions (radiation, high temperature, humidity). In the case of surface films such as contacts, their electical characteristics modulate potential and electric field in the surface area, controlling in that way transport mechanisms between the surface and bulk area. This is particularly important for photodetectors and metal semiconductor barriers including contacts.

Beside 1/f noise, burst noise could also induce discreate fluctuations of current between two or more levels. This type of noise is considered the most limiting factor in the performance of photodetectors. The origin of this noise, as well as its appearance in different voltage regions depends on the type of polarization and on the type of actual device, but it is usually ascribed to the presence of the defects in crystal lattice such as dislocations. Since burst noise is manifested in the presence of the so called excess current, investigations of its origin and factors that influence its amplitude could lead to better understanding of the burst noise. It was supposed that current flowing through the defects is modulated by the change in the charge state of the generation-recombination (GR) centers located near defects in the space charge region. When such GR center captures electron, local increase of the barrier height occurs. Electron flux passing through the barrier in the vicinity of GR center decrease, as a consequence of the barrier height increase, modulating (decreasing) excess current. Vice versa, emmision of the charge carrier from the trap center leads to the local decrease of the barrier height, thus increasing the excess current. Depending on the type of the device, this phenomenon could occur on the surface of the device also.

Amplitude of the excess current (i.e. burst noise) $\Delta I_{F}$, depends on the applied voltage $V_{F}$ in the exponential manner:

$$
\Delta \mathrm{I}_{\mathrm{F}}=\Delta I_{\text {sat }} \exp \frac{q V_{F}}{n_{B N} k T}
$$

where $\Delta I_{s a t}$ and $n_{B N}$, are saturation current and ideality factor, respectively. Since in this model current $\Delta I_{F}$ is considered to be generation-recombination current, $n_{B N}>1$, and depends on the lifetime and concentration of the charge carriers, recombination probability, thermal velocity of the electrons, etc. 


\subsubsection{Minimization of $1 / \mathrm{f}$ noise in silicides by ion implantation}

Both burst noise and $1 / \mathrm{f}$ noise are considered to be especially important in contact layers, so a large number of investigations are based on measurements of these type of noises in contacts, for example, silicides. Silicides belong to a very promising group of materials with low resistivity and good temperature stability that are used for fabrication of reliable and reproducible contacts. Investigations of this type of contacts include both their experimental development and the development of methods for their characterization such as noise level measurements and RBS analysis. The noise level measurements enable the control of the noise, which is important characteristic of metal-semiconductor electrical contacts (especially $1 / \mathrm{f}$ noise). Surface effects such as surface recombination fluctuations in carrier mobility, concentration of surface states, etc., have great influence on frequency dependent noise in silicides. Many authors in their investigation of silicides discuss the problem related to the application of ion beam mixing of As+ ions for the formation of silicides (Stojanovic et al., 1996a). Although introduction of As+ ions and their diffusion could change impurity concentration resulting in an increased noise level (especially for $1 / \mathrm{f}$ noise) in the structure, carefull optimization of the implantation dose and subsequent thermal treatment such as annealing could result in the formation of stable contacts with a low noise level. Every step of the silicides fabrication including preparation of Si substartes, deposition of metal layer (Pd, Ti, TiN, for example), As+ ion implantation, and annealing must be taking into consideration in order to achieve the best possible result. It has been found (Stojanovic et al., 1996a, 1996b) that both the implantation dose and ion energy, and annealing have a pronounced influence on the noise level in silicides. When Pd silicides are concerned, structural RBS analysis have shown that $\mathrm{Pd}_{2} \mathrm{Si}$ phase, which was already formed during preimplantation annealing is not affected by ion implantation. The spectra exibit slight changes in the slopes of the signal, corresponding to the $\mathrm{Pd}_{2} \mathrm{Si}-\mathrm{Si}$ interface. These changes can be attributed to additional intermixing of silicon and palladium atoms due to the presence of radiation-induced defects. It was also found (Stojanovic et al., 1996a) that the sequence of the fabrication steps influence the noise level in the formed silicides. The noise level was lowest in the samples implanted after annealing, so optimized ion implantation concerning implantation energy and dose, and average projected range of As+ ions near Pd-Si interface, does not reorder the silicides formed by annealing (also shown by RBS analysis), and induce more homogenous silicide/silicon interface. This suggests that thermal treatment induce relaxation of crystal lattice and improvement of the crystal structure of the silicides. On the other hand, noise measurements have confirmed that a more homogenous structure of Pdsilicides results in a lower frequency noise level.

The influence of the implantation dose on the noise level could clearly be seen for the Ti-TiN silicides example (Vasic et al., 2011). Structural RBS analysis has shown that ion implantation did not induce redistribution of components for lower implantation doses (Fig. 1). The spectra indicate that the entire titanium layer has interdiffused with the silicon substrate.

The presence of the $\mathrm{TiSi}_{2}$ and $\mathrm{TiSi}_{2}$ phase in the implanted samples was observed. In all cases top TiN layer remains unaffected, but for higher doses of implantation $\left(1 \times 10^{16}\right.$ ions $/ \mathrm{cm}^{2}$ ) a disordered structure was registered. This corresponds to the amorphization of silicon substrate, which is moving deeper with the ion dose, showing that the physical properties of TiN/Ti/Si are influenced by the implantation. 


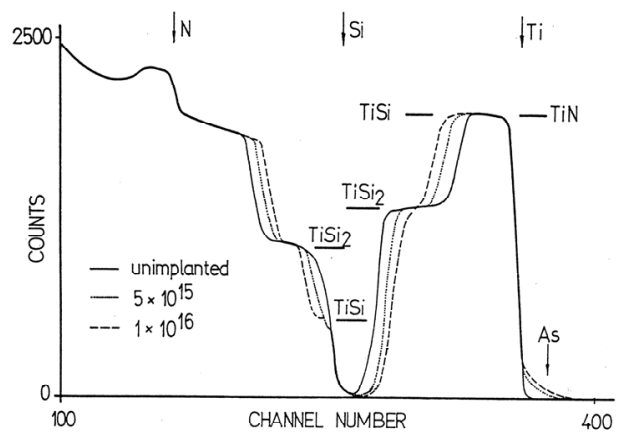

Fig. 1. RBS spectra of TiN/Ti/Si samples (Vasic et al., 2011).

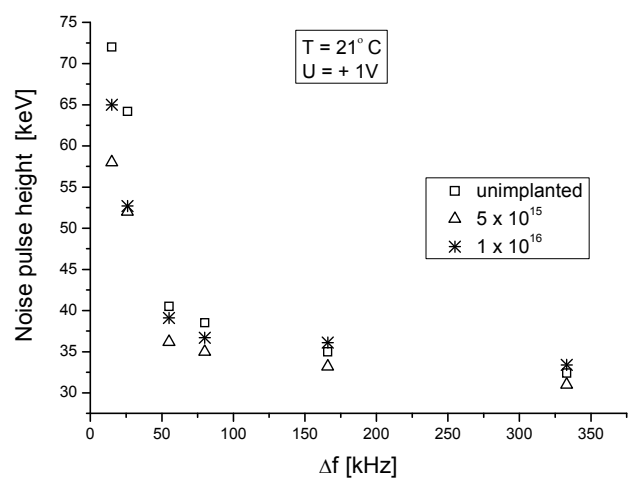

Fig. 2. Frequency noise level for TiN/Ti/Si samples at $21^{\circ} \mathrm{C}$ (Vasic et al., 2011).

This influence was confirmed by noise level measurements (all of the samples exhibit similar behaviour). Noise spectra were measured for different time constants $\tau$ (frequency range $\tau \sim 1 / f)$ of the low noise amplifier. Analysis of the results of these measurements shows that implantation could have influence on the noise level (Fig.2), but the main effect depends on the implantation dose. As discussed above, implantation dose of $1 \times 10^{16}$ ions $/ \mathrm{cm}^{2}$ induces some disorder in the structure that could lead to higher noise level. Ion dose of $5 \times 10^{15}$ ions $/ \mathrm{cm}^{2}$ shows the best results for the entire measuring range, suggesting that this dose of implantation induce a more homogeneous silicidation and the formation of Ti-Si phase with a lower concentration of crystal defects (after annealing). The lower concentration of point defects and dislocations and a more homogeneous silicide/silicon interface result in a lower frequency noise level of the analyzed structures.

\subsection{I-V measurements and the ideality factor}

Another commonly used and relatively simple method for obtaining output characteristics of photodetectors and solar cells related to the transport processes is current-voltage $(I-V)$ measurement. Any deviation of the transport mechanism from the ideal model of thermionic emission directly reflexes on the shape of current-voltage characteristics. Main 
parameter that could be extracted from $I-V$ data is the ideality factor $(n)$, direct indicator of the output parameter dependence on the electrical transport properties of the junction. Output characteristic of all semiconductor devices are primarily defined by fundamental parameters (resistance, lifetime and mobility of charge carriers, diffusion length etc.), and processes in them. Analytical connections between fundamental and output characteristics of solar cells are matter of theoretical analysis, but experimentally obtained results are more complex than theoretical suppositions. Both in production process of solar cells, and during their performance, the distribution of dopants, impurities and especially defects is usually not uniform and predictable, and could directly influence the processes in the cells. Factors that influence internal parameters of solar cells such as series and parallel resistance lead to changes in efficiency and maximum generated power in solar cell. Capability of solar cell to convert solar energy into electrical, depends on various fundamental and technological parameters (Stojanovic et al., 1998). Empirically obtained influence of fundamental parameters is usually mathematically defined by formal introduction of the ideality factor, $n$, in the exponent of current - voltage characteristics of solar cells. Ideality factor combines all variations of current flow from the ideal case, induced by various internal and external influences of physical parameters during the manufacturing process or as a consequence of aging. The non-ideal behavior of the device is reflected in the values of $n$ greater than 1 , and that is the result of the presence of different transport mechanisms that can contribute to the diode current. Determination of the dominant current mechanism is very difficult because the relative magnitude of these components depends on various parameters, such as density of the interface states, concentration of the impurities and defects, height of the potential barrier, device voltage, and device temperature (Vasic et al., 2004). Obtaining of the ideality factor from $I-V$ measurements is simple, non-invasive and effective way to evaluate possible degradation of output characteristics of solar cells and photodetectors in general in working conditions.

\subsubsection{Extraction of parameters from $I-V$ measurements}

Characteristic parameters of the semiconducting devices are often very difficult to determine and their values could depend on the used measurement methods. Currentvoltage measurements are widely used to characterize the barrier height, carrier transport mechanisms and interface states both in Schotky barriers and p-n junctions. This relatively simple method provides reliable and reproducible results, but the extraction of the diode parameters could be influenced by their voltage dependence and the presence of series resistance (Vasic et al., 2000, 2005).

In an ideal case of thermionic emmision as a dominant trasport mechanism of charge carriers, current flow across the junction diode under forward bias and in the case when $V-I R_{s}>>3 k T / q$, is usually represented by the equation:

$$
I=I_{s}\left[\exp \frac{q\left(V-I R_{s}\right)}{n k T}\right]
$$

where $I$ is diode current, $I_{s}$ the saturation current, $V$ the applied bias voltage, $R_{s}$ the series resistance, $n$ the ideality factor, $T$ the temperature, $q$ the electron charge and $k$ the Boltzman constant. The saturation current $I_{s}$ depends on the carrier transport mechanism across the junction, and the formally introduced ideality factor $n$ reflects the influence of various parameters (presence of the interface states, generation-recombination current, tunneling, 
spatial inhomogenities, etc.), and frequently is a function of the applied voltage (Vasic et al., 2005). Parameters $n$ and $I_{s}$ are direct indicators of the output characteristics of the semiconducting devices on the electric transport processes in the junction. Deviations of the ideal (Shockley) case of the carrier transport are mostly the result of the combined influence of following factors: surface effects, generation and recombination of the cerriers in the depletion region, tunneling between states in the energy gap, series resistance, etc. For homo-junctions with homogenous distribution of recombination centers, two limiting values for the ideality factor are usually taken into consideration: $n=1$ for the injection and diffusion in the depletion layer, and $n=2$ in the case of the domination of the generation-recombination current in the depletion layer (due to the recombination of the electron-hole pairs in the recombination centers - traps). Very rarely values of the ideality factor are exactly 1 or 2, and they usually depend on the applied voltage also. Variations from the predicted values are the consequence of many factors. On the microscopic level impurities and defects induced during manufacture could form regions of low carrier lifetime, and that could have influence on the $I-V$ characteristics, and hence the ideality factor also. Beside that, surface states could act as a recombination centers as well, and in that case ideality factor depends on the energy states of that recombination centers. If the space distribution of the recombination centers within the depletion region is nonuniform, the ideality factor could have values greater than 2 .

All of these factors make the extraction of the diode parameters very complicated since, usually, the first step in extraction of the diode parameters is the linear approximation of the $\ln I-V$ plot. Determination of the ideality factor value could be used as a measure of the validity of such a method. Namely, if the value of $n$ is approximately 1 , such approximation could be considered accurate enough, but if $n>1.4$, transport mechanisms that produce the deviation from the ideal model and non-linearity of the $\ln I-V$ plot should also be considered. Even when the type of the transport mechanism could be more or less preciselly determined, series and parallel resistance of the device should also be taken into consideration. Introduction of these parameters in the $I-V$ characteristic (eq.2) gives following dependence:

$$
I=I_{s 1}\left[\exp \frac{q\left(V-I R_{s}\right)}{k T}-1\right]+I_{s n}\left[\exp \frac{q\left(V-I R_{s}\right)}{n k T}-1\right]+\frac{V-I R_{s}}{R_{s h}}
$$

In this case, $\ln I-V$ plot is clearly not linear, because depending on the applied voltage, there could exsist one or more linear regions. The decrease of the $\ln I-V$ slope, i.e. increase of the ideality factor for lower voltage is a result of the presence of parallel resistance, $R_{s h}$ (due to "leakage" current across the junction). The same effect on the values of the ideality factor has series resistance, $R_{s}$ for higher voltages.

Since in the real semiconductiong device (solar cell, photodetector) many unpredictable factors (concentration of defects and impurities, exsistance of the energy states in the forbidden zone, etc.) influence their parameters $\left(n, I_{s}, R_{s}\right)$, determination of the ideality factor as a macroscopic quantity, could give accurate information about the quality of each device. The basis of most of methods for obtaining the diode parameters from $I-V$ curves (Vasic et al., 2005) is the correction of the experimentaly obtained data due to the presence of the series resistance $R_{s}$, but some of them also treat the diode parameters (especially $n$ and $I_{s}$ ) as voltage dependent. Analysis of the linear part of $\ln I$ vs $V$ plot and fitting of the experimental results is used in the so called numerical methods, whereas in other methods auxiliary functions based on some physical parameters are introduced. 


\section{Standard linearization method}

This method is based on the analysis of the linear part of $\ln I=f(V)$ plot using equation (2) when diode parameters are voltage independent $-I_{s}$ is calculated from the extrapolated intercept with the y (current) axis, and $n$ is deduced from the slope. However, when measured plot deviates from straight line shape (in the presence of $R_{s}$ ), calculated parameters are significantly different from the values obtained using other methods. This is very distinct when $R_{s}$ is high and the shape of the $I-V$ curve is not the same in different voltage regions (especially for high forward bias).

\section{Numerical method}

Enhanced version of the standard method also includes presence of the series reistance, but the diode parameters could be determined without linearization of the $I-V$ curve (Vasic et al., 2005). Method is based on a least square method of fitting the experimentally obtained results in order to yield optimal values of $I_{s}, n$, and $R_{s}$.

When an applied voltage $V_{A}$ is provided across the device terminals, actual barrier (junction) voltage $V$ is given by:

$$
V=V_{A}-I_{E} R_{S}
$$

( $I_{E}$ is experimentally measured diode current). Then, from the equation (2) and using equation (4), applied voltage is given by:

$$
V_{A}=I_{E} R_{s}+a \ln I_{E}+b
$$

where:

$$
a=n k T / q \text { and } b=-a \ln I_{s}
$$

Thus if $R_{s}, a$, and $b$ could be determined, then $n$ and $I_{s}$ could also be obtained. One of the ways for optimization of $R_{s}, a$, and $b$ is method of least squares, and for this purpose function $S$ is defined as:

$$
S=\sum_{i=1}^{m}\left\{I_{E i} R_{s}+a \ln I_{E i}+b-V_{E i}\right\}^{2}
$$

where $m$ is the number of diode pairs of experimentally determined $I_{E}$ and $V_{E}$. The optimal combination of parameters would result in a minimum value of $S$, and from the set of matrix equations values of $R_{s}, a$, and $b$ could be calculated. Then, using equation (6) $n$ and $I_{s}$ are easilly obtained.

It has been shown (Vasic et al., 2005) that the agreement between experimental data and numerical simulation is very good, especially when parasitic series resistance effects are present, and when the measured $\ln I=\mathrm{f}(V)$ plot shows no linear regime. However, since this method is not based on any physical model of current transport, there are no sufficient indications of the validity of these results.

\section{Methods based on the physical models}

Physical parameters such as differential conductance and resistance, for example, are the basis of some methods that use auxiliary functions or parameters for the determination of real diode parameters. Novel methods take into account that for the real diodes $n \neq 1$, and 
also the fact that at high bias voltages series resistance could significantly influence the shape of the $I-V$ plot, making the linear fit for the whole voltage region impossible. Nevertheless, these methods usually treat such problems separately. Namely, most of the methods do not take into account that the diode parameters $\left(n, I_{s}\right)$ are voltage-dependent. On the other hand, methods that analyze voltage-dependent parameters produce correct results only under assumption that the influence of the series resistance is negligible. However, generally speaking, for real diodes neither series resistance nor voltage dependence are negligible, so most of the proposed methods have limited application and give unreliable results.

There are several approaches for determination of the voltage-dependent ideality factor:

1. differentiation of the simplified expression of equation (2):

$$
\frac{1}{n(V)}=\frac{k T}{q} \frac{\partial}{\partial V}(\ln I)
$$

but numerical differentiation is, in effect, a total (complete) and not partial differentiation, so the obtained result is some function $\mathrm{f}(n)$, and not the true ideality factor $n(V)$,

2. calculation of the function $n(V)$ at each point of the experimental $I-V$ data (method "point by point") using equation:

$$
n(V)=\frac{q V}{k T} \ln \left(\frac{I}{I_{s}}+1\right)
$$

with the assumption that the saturation current is previously determined in accordance with the influence of the series resistance,

3. method of adding an external resistance, based on two consecutive measurements:

a. conventional $I-V$ measurements, and

b. $\quad I-V$ measurement with external resistance $R_{e x}$ in series with the diode.

From the equation (2) and after adding $R_{e x}$, following set of equations were obtained:

$$
\begin{gathered}
\ln I=\ln I_{s}+\frac{q}{n(V) k T}\left(V-I R_{s}\right) \\
\ln (I+\Delta I)=\ln I_{s}+\frac{q}{n(V) k T}\left[V-(I+\Delta I)\left(R_{s}+R_{e x}\right)\right]
\end{gathered}
$$

where $\Delta I$ is the change in $I$ produced by adding $R_{e x}$. From those equations follows:

$$
\frac{\Delta(\ln I)}{R_{e x}}=-\frac{q}{n(V) k T}\left[\left(R_{s}+R_{e x}\right) \frac{\Delta I}{R_{e x}}+I\right]
$$

Equations (10) and (12) form the system of two equations with three unknown diode parameters $\left(R_{s}, I_{s}\right.$ and $\left.n\right)$, so some relationship must be established between any two of the unknown parameters. There are two methods of eliminating one unknown parameter; first method ("A") is based on establishing the relationship between the ideality factor and 
saturation current by eliminating series resistance from the equation (10), and obtaining from the equation (11):

$$
\begin{gathered}
f_{2}(I, \Delta I)=\ln I_{s}+\frac{q}{n(V) k T} f_{1}\left(V, I, \Delta I, R_{e x}\right) \\
f_{2}(I, \Delta I)=\ln I-\frac{\Delta I}{I} \ln \left(1+\frac{\Delta I}{I}\right) \quad \text { and } \quad f_{1}\left(V, I, \Delta I, R_{e x}\right)=V+R_{e x} I\left(1+\frac{I}{\Delta I}\right)
\end{gathered}
$$

Using the $\mathrm{f}_{2}=\mathrm{f}\left(\mathrm{f}_{1}\right)$ dependence, saturation current $I_{s}$ and ideality factor $n$ could be obtained from the slope and the vertical axis intercept of the plot, independently of the series resistance ( $R_{s}$ could be calculated from the equation (2), afterwards).

However, in the case of voltage-dependent $n$, dependence $f_{2}=f\left(f_{1}\right)$ is not a straight line, so second method ("B") that allows determination of all parameters in the case of non-linearity, must be used. The ideality factor $n$ is, at the beginning, eliminated from the equations (11) and (12), thus establishing the relationship between $I_{s}$ and $R_{s}$ :

$$
R_{s}=-\frac{1}{\Delta I}\left[\frac{f_{1}}{f_{2}-\ln I_{s}} \ln \left(1+\frac{\Delta I}{I}\right)+R_{e x}(I+\Delta I)\right]
$$

assuming that both $I_{s}$ and $R_{s}$ are voltage independent over the range of interest. Saturation current could be determined by fitting in such a way that series resistance would have approximately constant value. The obtained values of $I_{s}$ and $R_{s}$ could then be used to extract the voltage-dependent $n(V)$ from the equation (2).

It was shown that both of these methods that use the auxiliary function $\mathrm{f}_{1}$ and $\mathrm{f}_{2}$ give reliable and reproducible results. First one ("A") is limited on the cases of voltage independent parameters, but gives good results in the presence of noise, while method " $\mathrm{B}$ " provides the possibility of analyzing the general case of voltage-dependent ideality factor in the presence of series resistance and experimental noise.

\section{Verification of the presented methods}

The validity of the above-described methods was examined by analyzing $I-V$ characteristics (primarily obtaining the values of the ideality factor) of real photodiodes (Vasic et al., 2005). Samples used in the experiment were commercially available $p-i-n$ and $p-n$ silicon photodiodes (trademarks BPW 34, BPW 43, SFH 205, and BP 104). Forward bias dark $I-V$ characteristics of the diodes were measured using standard configuration for the $I-V$ measurements. In all samples presence of the series resistance was detected (calculated range of $R_{s}$ was from $20 \div 30 \Omega$ for BPW 34 and SFH 205 p-i-n type, to $100 \div 200 \Omega$ for BPW 43 p-n type of photodiodes). Values of the ideality factor calculated using standard method were in the range of $1.2 \div 1.6$, depending on the type of the samples. Similar range of values was obtained by numerical method, while application of the auxiliary functions (method "A") results in lower values of $n(1.05 \div 1.5)$. Considering physical meaning of the ideality factor (measure of non-ideality of the junction), the only way to verify the validity of a given method is evaluation of the agreement between experimentally measured data and numerically calculated $I-V$ curves (with calculated diode parameters), i.e., determination of the correlation factor $r$. For standard and numerical method correlation factor was higher than 0.99 , but the best values of $r$ were for the method "A". Figure 3 shows agreement for 
standard method and method "A" for one sample, 3 BPW 43 (dots represent experimental data, and lines - calculated characteristics). Correlation factor for all three used methods was good $(r>0.99)$, so the choice of the exact method depends on the complexity of the procedure and experiment requirements.

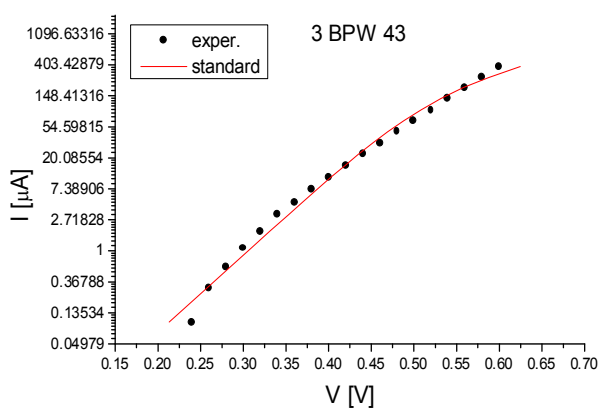

a)

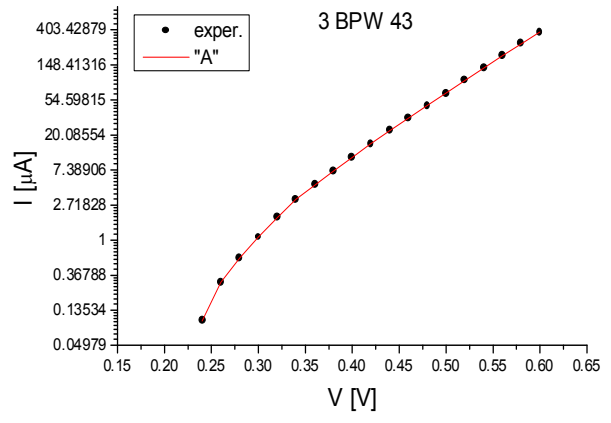

b)

Fig. 3. Agreement: experimental and calculated data for a) standard, b) method "A" (Vasic et al., 2005).

In order to test the assumption that the ideality factor is voltage-independent and that the determination of only one, "summary" ideality factor is sufficient, diagrams of $f_{2}=f\left(f_{1}\right)$ dependence were plotted (two characteristic examples are shown in Fig. 4). Correlation factor for those functions for the sample 3 BPW 43 was the highest $(r=0.99751)$, and that, together with the obvious good linearity of the $f_{2}=f\left(f_{1}\right)$ diagram, shows that for this sample ideality factor has approximately constant value for whole voltage range. However, although the agreement between experimental and calculated data for both shown samples were good, from Fig. $4 b$ ) could be seen that $f_{2}=f\left(f_{1}\right)$ dependence for sample 9 BPW 34 was not linear, so junction parameters are voltage dependent. This makes the determination of the local ideality factor $n_{l o c}$ (i.e., determination of the $n(V)$ dependence) necessary.

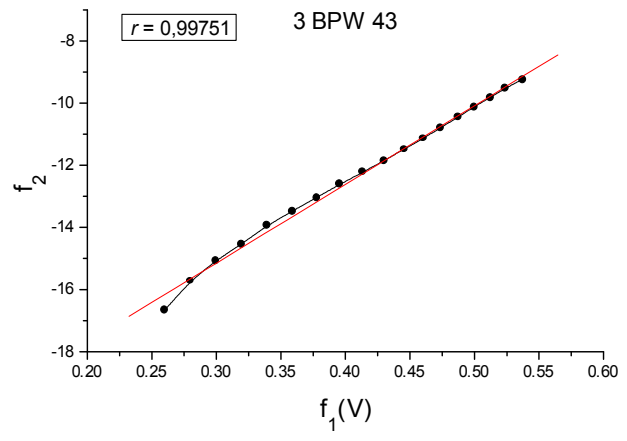

a)

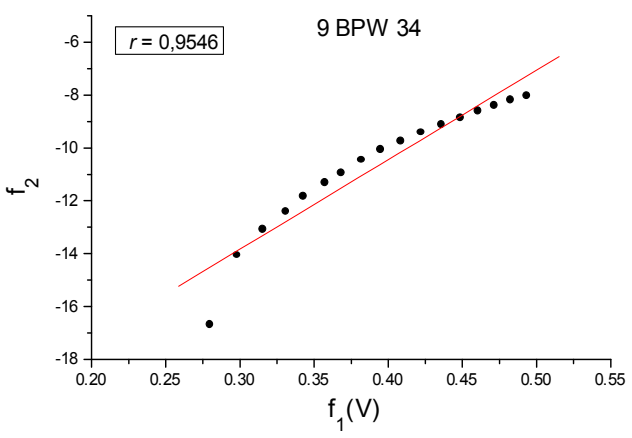

b)

Fig. 4. Diagram of the $f_{2}=f\left(f_{1}\right)$ dependence for samples a) 3 BPW 43 and b) 9 BPW 34 (Vasic et al., 2005). 
Differentiation of the equation (2) leads to as previously explained, some function $\mathrm{f}(n)$, due to the presence of $R s$ :

$$
f(n)=\frac{k T}{q} \frac{d}{d V}(\ln I)=\frac{1}{n}-\frac{V}{n^{2}} \frac{d n}{d V}
$$

Except in cases when $\mathrm{d} n / \mathrm{d} V<<n(V) / V$ at the same bias, second term in the right hand side of the equation (15) cannot be neglected. Therefore, the exact values of the ideality factor could be obtained by integration of the equation (15), which is difficult, due to the unknown initial conditions.

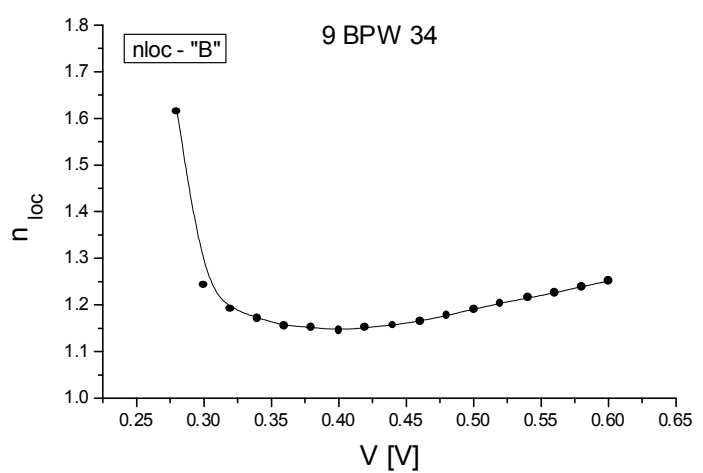

Fig. 5. Method "B" (Vasic et al, 2005).

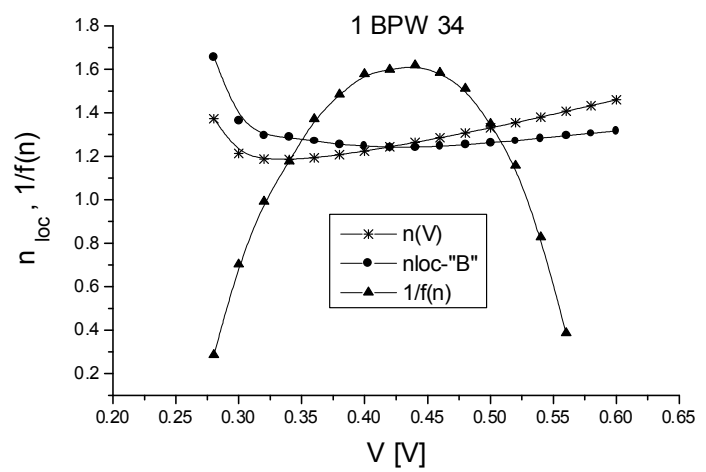

Fig. 6. Comparison for $n_{l o c}$ (Vasic et al., 2005).

Above-mentioned deviations of the $f_{2}=f\left(f_{1}\right)$ plot from the linearity are ascribed to the bias dependence of the ideality factor. Reliable and accurate method for obtaining the local ideality factor directly, is method "B". For the sample 9 BPW $34\left(f_{2}=f\left(f_{1}\right)\right.$ significantly deviates from the linearity, Fig. 4 b)), bias dependent $n_{l o c}$ was calculated using proposed method, and the result is shown in Fig. 5. From this figure it could be seen that the ideality factor is constant only in the very narrow voltage region (from 0.32 to $0.42 \mathrm{~V}$ ). The correlation factor for method "B" was very good (near 1). However, beside very high 
precision of the obtained results, the complexity of the method presents in some cases, the major limiting factor for its application. Adding an external resistance, together with the problem of selection of an optimal value for $R_{\text {ex }}$ makes these methods (both " $A$ " and "B") inappropriate in the field conditions for example, or when a quick evaluation of the $I-V$ data is necessary. In this case, method based on the calculation of the $n(V)$ at each point of the experimental $I-V$ data - method "point by point", should be used (equation (9)). In Fig. 6, bias dependent ideality factor calculated using method " $B$ " (filled circles) and "point by point" (asterisk) for one sample (1 BPW 34) is presented. It could be seen that both methods give similar values of $n$, as well as the same $n(V)$ behavior (high values of $n_{l o c}$ at low bias, decrease for medium, and slight increase for higher bias). For the comparison, in the same figure $1 / f(n)$ dependence was also plotted (triangles). The only condition that should be fulfilled for using equation (9) is to determine the value of the saturation current previously, and this could be done by any of the proposed methods.

The extraction of diode parameters is difficult due to the presence of the series resistance, and values of $I_{s}, n$, and $R_{s}$ usually depend on the methods used for their calculation. The choise of the exact method depends both on the complexity of the method and on the experimental demands (conditions). Methods of using auxiliary functions (methods " $\mathrm{A}$ " and " $\mathrm{B}$ ") demand adding (optimal) external resistance and additional measurements. Also, calculation of the diode parameters is more complex than for other methods due to the extended numerical procedure. In laboratory conditions, these methods undoubtedly give the best results, but in the field conditions both of these methods are very complex and the extraction of the parameters is slow. This is more emphasized if the devices are exposed to the severe working conditions when quick assessment of their performance is needed. Even in the laboratory conditions, when the relative change of the diode parameters is measured for the estimation of the "worst-case scenario", both standard and numerical method gave good results for voltage-independent parameters, and "point by point" method for voltagedependent parameters. Based on the experimental verification, it could be concluded that, although the results obtained by the methods of adding an external resistance have the best agreement with the experimental data, standard and numerical methods are more suitable for quick extraction of diode parameters.

\section{Radiation effects and possibilities of improvement of solar cells characteristics}

Beside the diversity of the device technologies used for designing of the optical and other detectors, there are a variety of radiation environments in which they are used (natural space and atmospheric, as well as military and civil nuclear environments, etc.). Reliability of electrical devices in a radiation environment is very important, and extensive studies concerning the development of semiconductor devices that can operate normally in such conditions have been seriously undertaken. Possible degradation of the electrical performance of solar cells and photodetectors induced by irradiation means that very strict conditions for their application must be predetermined for the worst case scenario. Performance failure in such conditions could have negative impact both on the financial and environmental aspects of the device application. Therefore, from the technological point of view, it is important to study the variations induced by irradiation of semiconductor 
junction characteristic parameters (ideality factor, saturation current, etc.), that affect the performance of the photodiodes and solar cells.

Investigations of radiation effects in solids are primarily based on the study of the characteristics based on structure. Since radiation unduced deffects are connetcted to the defects in the crystal lattice, physical evidence of such a damage must br found through those characteristics that are most sensitive to the unperfections of the lattice. Due to the microscopic nature of such a defects, measurements of variations in those charcateristics are almost the only method of evaluation of radiation influence (Loncar et al., 2005, 2006, 2007). In semiconductor devices, radiation induced defects are connected to the localized energy states that could change concentration and mobility of the charge carriers. Namely, the main charactersitics of semiconducting devices is precisely the change (in a large range) of the charge carriers concentration, weather because of the defects and impurities, or under the influence of the raised temperature. When the equilibrium concentration of electrons and holes is disturbed by the radiation, for example, their mutual anihillation is possible only in the localized energy states in the crystal. These are, so called, recombination centers, and radiation induced defects represent very good example of such a centers.

Interaction of radiation with the semiconducting devices basically produses three effects: permenent ionization damage in insulator layers, ionization effects in semiconductors, and dislocation damage. During interction of $\gamma$ radiarion with the device, and depending on the radiation energy, primarilly three effects could occur: photoelectric effect, Compton effect, and electron-hole creation. In all of these processe, absorbed photon energy couses ionization and excitation of the created electrons, with the electron-hole creation. These effects lead to the generation of parasitic charge (noise) collected in the depletion region. Also, ionization effects could produce recombination centers in the energy gap. In silicon, all of these effects could be permenent or quasi-permenent.

On the other hand, dislocation damage usually arise after the interaction of the particled such as protons, neutrons and electrons. Processes that could happen during those collisions depend on the type of particles and their energy. Collision of the particles with the atoms of crystal lattice could produce vacances, interstities, and other types of defects. These defects could be traped in the impurity centers, for example, and could form active defects leading to the changes in the characteristics of the photodetectros, primarilly the decrease of the minority carrier lifetime. Radiation damage due to neutrons (heavy particles) is, as mentioned above, primarily connected to the displacement of silicon atoms from their lattice sites in the crystalline silicon solar cells, leading to destruction and distortion of local lattice structure and formation of defects. If, under the influence of neutrons, stable defects are made, they could, together with impurity atoms, donors and for example implanted atoms, form complex defects acting as recombination sites or traps, significantly decreasing minority carrier lifetime.

\subsection{Radiation damage in solar cells and photodiodes}

The main effect of radiation on photodiodes and solar cells is an increase in the dark current generated within or at the surface of the depletion region. Generation of electron-hole pairs due to ionization effects and displacement damage induced by neutrons, result in the generation and increase of the noise and the minimum signal that can be detected. Since presence of the noise could be connected to the excess current, measurement of the $I-V$ 
characteristics before and after irradiation reveals the extent of degradation of electrical properties of photodiodes.

Although polycrystalline and monocrystalline solar cells are more reliable than amorphous, inherent presence of defects and impurities in the basic material could, during time, produce some negative effects. This is specially emphasized if those states are located within the energy gap and are activated during work. In such a case they become traps for optically produced electron-hole pairs, and thus decrease the number of collected charge carriers. Macroscopically, this effect could be observed as a decrease of the output current and voltage, and ultimately could lead to the decrease of the efficiency of solar cell. Lower values of short-circuit current indicate the existence of the recombination centers that decrease the mobility and diffusion length of the charge carriers, making the recombination in the depletion region dominant transport in such solar cells (Vasic et al., 2000, 2003). The lifetime of the solar cell is restricted by the degree of radiation damage that the cell receives. This is an important factor that affects the performance of the solar cell in practical applications. The permanent damage in the solar cells materials is caused by collisions of the incident radiation particles with the atoms in the crystalline lattice, which are displaced from their positions. These defects degrade the transport properties of the material and particularly the minority carrier lifetime (Alurralde et al., 2004, Horiushi et al., 2000, Zhenyu et al., 2004). This lifetime decrease produces degradation of the parameters of the cell ultimately leading to an increase of the noise level. The interaction between vacancies, selfinterstitials, impurities, and dopants in Si leads to the formation of undesirable point defects such as recombination and compensator centers which affects performance of the solar cells, especially in space. Introduction of radiation-induced recombination centers reduce the minority carrier lifetime in the base layer of the $p-n$ junction increasing series resistance. After very high doses of radiation series resistance of the base layer could be so high that most of the power generated by the device is dissipated by its own internal resistance (Khan et al., 2003).

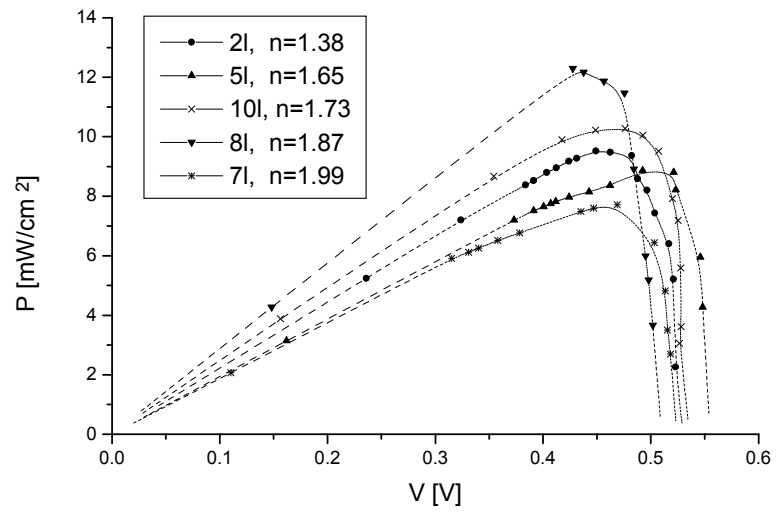

Fig. 7. Simulation of the dependence of $P$ - $V$ characteristics on $n$ (Vasic et al., 2011). 
Capability of solar cell to convert solar energy into electrical, depends on various fundamental and technological parameters. Variations from the ideal case of current transport could be represented by the ideality factor that could be easily obtained from $I-V$ characteristics of solar cells. The non-ideal behaviour of the device is reflected in the values of $n$ greater than 1, and that is the result of the presence of different transport mechanisms that can contribute to the diode current.

Determination of the dominant current mechanism is very difficult because the relative magnitude of these components depends on various parameters, such as density of the interface states, concentration of the impurities and defects, height of the potential barrier, device voltage, and device temperature. The dependence of the maximum power on the ideality factor could be seen in Fig. 7.

Considering the fact that maximum power point depends on the resistance (and ideality factor as well), series and parallel resistance should be maintained at such a values to obtain maximum efficiency. Also, voltage decrease in the maximum power point $\left(P_{m}\right)$ has great influence on the efficiency. One of the main reasons for this decrease is the increase of the ideality factor, so it could be said that the influence of the ideality factor on the solar cell efficiency is through the voltage. Set of the experimentally obtained $\eta=\mathrm{f}(n)$ dependencies for different solar cells is shown in Fig. 8 (Vasic et al., 2000).

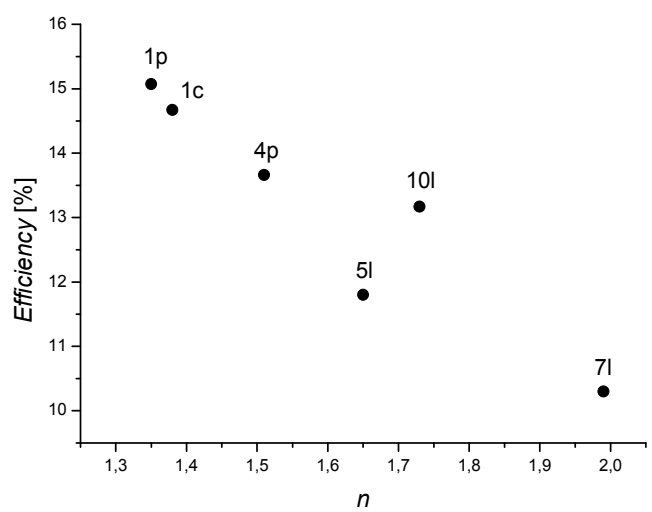

Fig. 8. Efficiency dependence on the ideality factor (Vasic et al., 2000).

Regardless to the type of radiation used, damage to even a small portion of the individual solar cell results in an increase in saturation (leakage) current for whole cell. The cell diode saturation current (or more commonly used its density) $J_{0}$, increases with the decreased minority carrier lifetime. On the other hand, the minority carrier lifetime decreases due to the ionization effects and displacement damage in the depletion region, caused by the incident radiation. In consequence, this reduces the cell open circuit voltage $V_{o c}$. Since forward-biased cell diode current increases for all diode voltages, the current available to the load decreases, so that the maximum power delivered to the load, $P_{\max }$, will also decrease, leading to the substantial drop of the cell efficiency $\eta$. Such non-ideal behavior of the device is usually reflected in the values of the ideality factor $n$ greater than 1 , as the result of the presence of different transport mechanisms in different voltage regions that can 
contribute to the diode current. Since the ideality factor is the direct indicator of the output parameter dependence on the electrical transport properties, measurements of the $n(V)$ dependence along with the $I-V$ measurements at different irradiation doses, could narrow down possibilities of the dominant current component. Also, values of the ideality factor could indicate not only the transport mechanism, but indirectly, the presence and possible activation of the defects and impurities, acting as recombination and/or tunneling centers.

The influence of the ideality factor on the solar cell efficiency is predominantly through the voltage, i.e. the decrease of the efficiency with the increase of the ideality factor is the result of the voltage decrease in the maximum power point. Physical basis of such dependence lies in the connection between the ideality factor and saturation current density shown in Fig. 9 (for different types of solar cells).

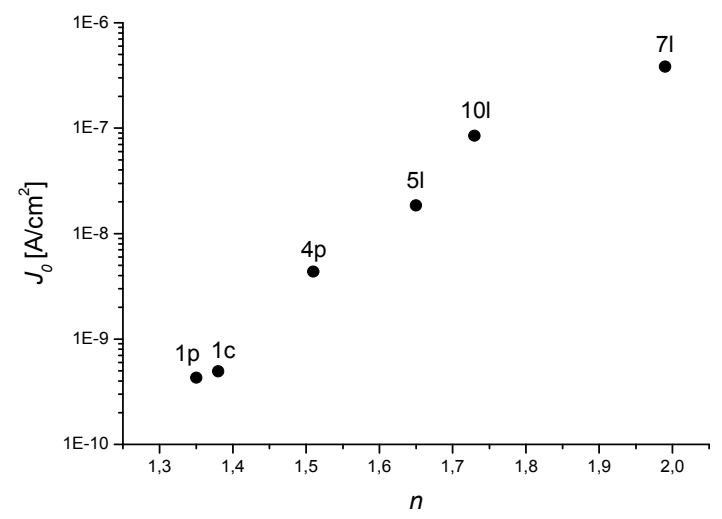

Fig. 9. Saturation current dencity dependence on ideality factor (Vasic et al., 2000).

Direct connection between $J_{0}$ and $n$ (nearly exponential increase of saturation current density with the increase of $n$ ) produces the decrease of the efficiency with the increase of either of these parameters. In the radiation environment, such an increase is usually the result of induced defects and/or activation of the existent impurities that could act as a recombination centers for the charge carriers, altering the dominant current transport.

Determination of the dominant current mechanism is very difficult because the relative magnitude of these components depend on various parameters such as, density of the interface states, concentration of the impurity defects, and also devises operating voltage. Existence of the $n(V)$ dependence is the result of such a junction imperfections, leading to domination of different transport mechanisms in different voltage regions. Therefore, measuring and monitoring the $n(V)$ dependence which is possible even in working conditions, could reveal not only the degree of degradation, but also, possible instabilities of the device in certain voltage regions. This is especially important if those instabilities occur in the voltage region where maximum power is transferred to the load. Although still in working condition, performances of such solar cells (efficiency, for most) are considerably degraded, so that monitoring of the device characteristics should be performed 
continuously, especially if solar cells are exposed to severe working conditions, such as radiation environment.

Although effects of gamma irradiation on the solar cells are known to be primarily through ionization effects, increase of series resistance could also be observed, Fig. 10 (Vasic et al., 2007, 2010).

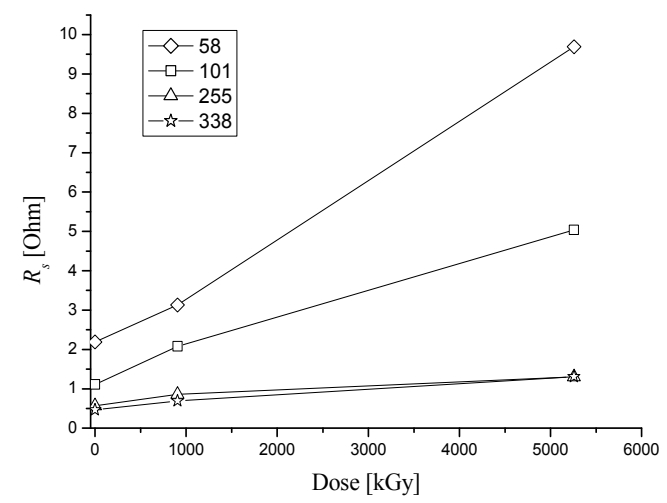

Fig. 10. Dependence of $R_{s}$ on doses for polycrystalline solar cells (Vasic et al., 2007).

Almost linear dependence of series resistance on the absorbed irradiation dose indicates that some changes in the collection of the charge carriers have occurred. This behaviour of $R_{s}$ is reflected mostly on the short-circuit current density $J_{s c}$, since radiation induced activation of defects and impurities mainly affects the transport mechanisms in the device. Dependence of the $J_{s c}$ on the absorbed dose for different illumination levels was shown in Fig. 11.

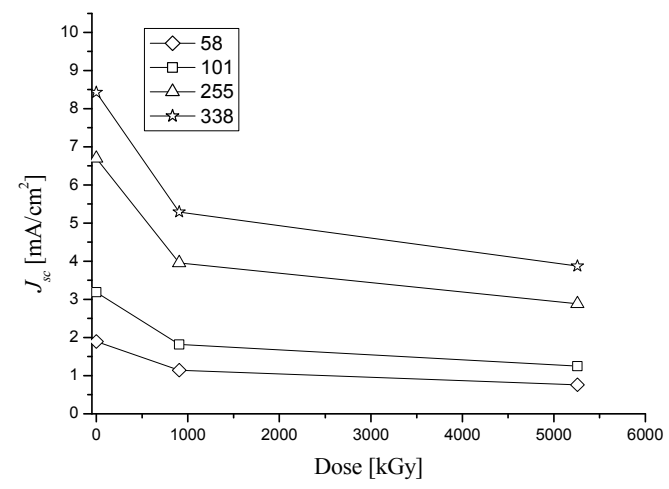

Fig. 11. Dependence of the $\mathrm{J}_{\mathrm{sc}}$ on doses for polycrystalline solar cells (Vasic et al., 2007).

Due to the inevitable presence of surface energy states (as a result of lattice defects, dislocations, impurities, etc.), after silicon is irradiated with gamma photons, both the surface recombination velocity and the density of surface states increase. If those states 
correspond to deep energy level in the silicon energy gap, they act as efficient surface recombination centers for charge carriers. Generation of electron-hole pairs due to ionization effects usually result in the generation and an increase of the noise and minimum signal that can be detected. All of these effects lead to the decrease of output current. Steeper decrease of the $J_{s c}$ for higher illumination levels indicates that recombination centers could be both optically activated and activated by irradiation. Therefore, solar cells exposed to the higher values of solar irradiation during their performance could exhibit greater decrease in the initial $J_{s c}$.

Additionally, if solar cells are polycrystalline, so presence of grain boundaries, characteristic for the polycrystalline material, has great influence on the collection of the photogenerated carriers. Presence of the recombination centers, small diffusion length and minority carrier lifetime, as a result of either irradiation or aging, finally leads to the decrease of the efficiency of solar cells. As could be seen in Fig. 12, this decrease is very pronounced, regardless of the illumination level. Although initial efficiencies were slightly different for different illumination levels, after irradiation they became almost equal, indicating that radiation gas greater influence on production and transport of charge carriers than illumination. That, from the standpoint of solar cells, could be very limiting factor for their performance. Combined influence of the increased $1 / \mathrm{f}$ and burst noise due to radiation induced damage has significant negative influence on major solar cells characteristics.

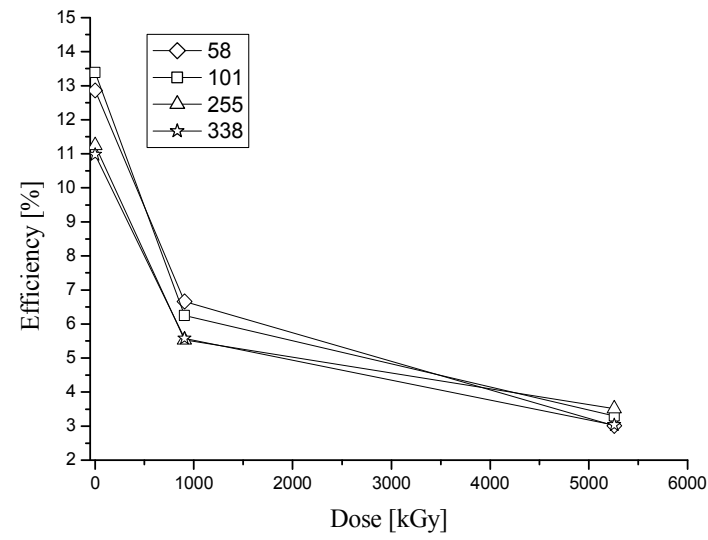

Fig. 12. Dependence of the efficiency on doses (Vasic et al., 2007).

All of this inevitably leads to the decrease of the resolution of the photodetector devices, lowering solar cells efficiency and for this reason, monitoring of the device characteristics should be performed continuously, especially when solar cells are exposed to the severe working conditions.

\subsection{Possibilities of the improvement of solar cells and photodetectors}

The lifetime decrease of the charge carriers due to the radiation damage induced by neutrons, produces degradation of electrical parameters of the cell, such as series resistance $\left(R_{s}\right)$, output current and finally efficiency $(\eta)$. High level of series resistance usually indicate 
the presence of impurity atoms and defects localized in the depletion region acting as traps for recombination or tunneling effects, increasing dark current of the cell (Alexander, 2003, Holwes-Siedle \& Adams, 2002). Moreover, shallow recombination centers in the vicinity of conducting zone enhance tunneling effect, further degrading output characteristics of the cell by increasing noise level (especially burst noise that is connected to the presence of excess current).

Such negative impact of neutron radiation was observed higher illumination level, as could be seen in Fig. 13 (Vasic et al., 2008). But interesting phenomena - the decrease of series resistance, was observed for lower values of illumination. (Different behavior for different illumination level is due to the presence of finite series and parallel resistance in the cell.) This decrease is very significant from the solar cell design standpoint because it indicates possible beneficent influence of low doses of irradiation, even with neutrons. It could be explained by the fact that during fabrication process of any semiconducting device, structural defects and impurities that were unavoidably made, produce tension in the crystal lattice. Low doses of radiation could act similarly to annealing, relaxing lattice structure and decreasing series resistance. Subsequently, this leads to lowering of noise level and an increase of the output current as shown in Fig.14 $\left(J_{m}\right.$ - current in the maximum power point).

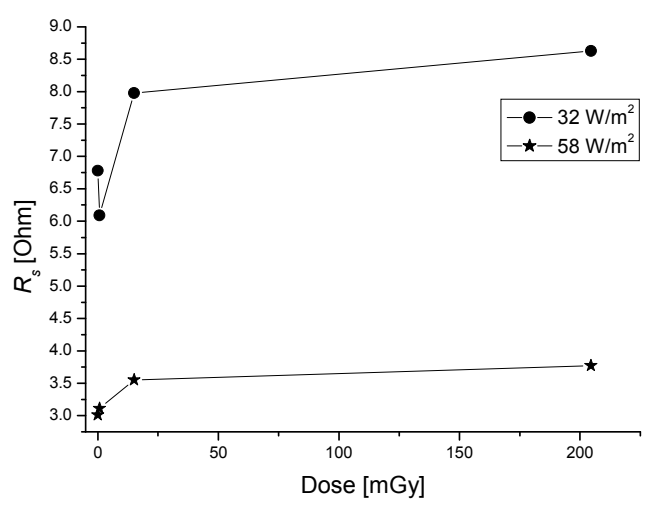

Fig. 13. Dependence of $R_{s}$ on doses for two illumination levels (Vasic et al., 2008).

Other parameters of solar cells (voltage in the maximum power point $V_{m}$, fill factor $f f$ and efficiency) have shown the similar tendencies, which is not surprising since, as it is well known, high series resistance of the solar cell is one of the main limiting factors of the efficiency. So, it could be expected that all the main output parameters of the solar cell should exhibit the same behavior as series resistance in the relation to the irradiation dose. Finally, improvement of output characteristics after the first irradiation step for low illumination level is registered for the efficiency also, Fig. 15. Although higher doses of neutron radiation undoubtedly have negative impact on the performance of solar cells, observed phenomena give possibilities for using radiation as a method for the improvement of solar cell characteristics. 


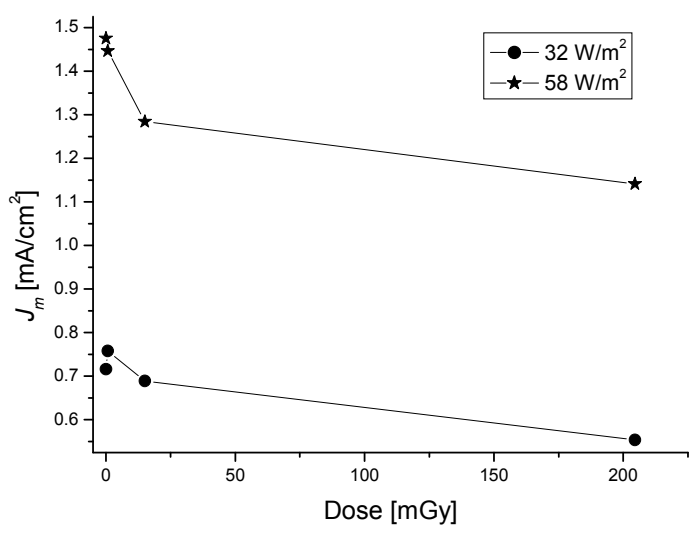

Fig. 14. Dependence of the $J_{m}$ on doses (Vasic et al., 2008).

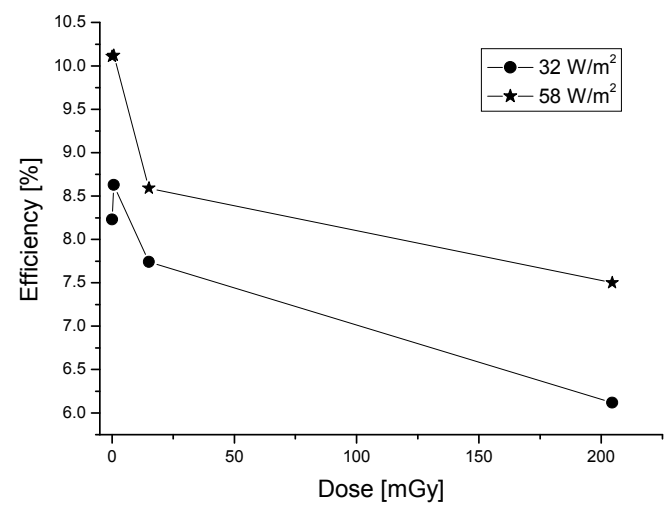

Fig. 15. Dependence of the efficiency on doses (Vasic et al., 2008).

Though commonly referred to as a source of noise in semiconducting devices, radiation induced effects (interaction of neutrons with Si solar cells, in particular) could have in some cases positive effect on main electrical characteristics $\left(R_{s}, J_{m}, \eta\right)$. Initial improvement of the characteristics observed for small doses of neutron radiation and low illumination level, indicates that there is a possibility of using irradiation for enhancement of the solar cells quality. 


\section{Conclusion}

Single element optical detectors such as solar cells and photodiodes are the final component needed for a communications or optical information processing systems. Due to wide area of application, they are often exposed to the variety of radiation effects (natural space environment, atmospheric environment, military and civil nuclear environment). Therefore, the extensive studies concerning the development of semiconductor devices that can operate normally in a radiation environment have been undertaken. Although proven to be reliable in terrestrial applications, solar systems are (like other semiconductor devices) sensitive to variety of radiation environments in which they are used. Performance failure could have negative impact both on the financial and environmental aspects of the device application. From a technological point of view, it is important to study the variations induced by irradiation of semiconductor junction characteristic parameters (reverse saturation current, ideality factor etc.), that affect the performance of the solar cells and photodiodes.

\section{Acknowledgment}

The Ministry of Science and Technological Development of the Republic of Serbia supported this work under contract 171007.

\section{References}

Alexander, D.R. (2003). Transient Ionizing Radiation Effects in Devices and Circuits, IEEE Transaction on Nuclear Sciences, Vol.50, No. 3, pp. 565-582, (2003), ISSN 00189499

Alurralde, M., Tamasi, M. J. L., Bruno, C. J., Martinez Bogado, M. G., Pla, J., Fernandez Vasquez, J., Duran, J., Shuff, J., Burlon, A. A., Stoliar, P., \& Kreiner, A. J. (2004). Experimental and theoretical radiation damage studies on crystalline silicon solar cells, Solar Energy Materials E Solar Cells, Vol. 82, pp.531-542, (2004), ISSN 0927-0248

Holwes-Siedle, A.G., \& Adams, L. (2002). Handbook of Radiation (Second Edition), Oxford University Press, ISBN13 9780198507338, Oxford

Horiushi, N., Nozaki, T., \& Chiba, A. (2000). Improvement in electrical performance of radiation-damaged silicon solar cells by annealing, Nuclear Instruments and Methods A, Vol. 443, pp. 186-193, (2000), ISSN 0168-9002

$\mathrm{Hu}, \mathrm{Z}$., He, S., \& Yang, D. (2004). Effects of $<200 \mathrm{keV}$ proton radiation on electric properties of silicon solar cells at 77 K, NIM B Beam Interaction with Materials \& Atoms, Vol. 217, pp. 321-326, (2004), ISSN 0168-583X

Jayaweera, P.V.V. , Pitigala, P.K.D.D.P., Perera, A.G.U., \& Tennakone, K. (2005). 1/f noise and dye-sensitized solar cells, Semiconductor Science Technology, Vol. 20, pp.L40-L42, (2005), ISSN 0268-1242

Jayaweera, P.V.V. , Pitigala, P.K.D.D.P., Senevirante, M.K.I., Perera, A.G.U, \& Tennakone, K. (2007). 1/f noise in dye-sensitized solar cells and NIR photon detectors, Infrared Physics \& Technology, Vol. 50, pp. 270-273, (2007), ISSN 1350-4495

Khan, A., Yamaguchi, M., Ohshita, Y., Dharmaraso, N., Araki, K., Khanh, V.T., Itoh, H., Ohshima, T., Imaizumi, M., \& Matsuda, S. (2003). Strategies for improving 
radiation tolerance of Si space solar cells, Solar Energy Materials \& Solar Cells, Vol.75, pp. 271-276, (2003), ISSN 0927-0248

Kovačević-Markov, K., Vasić, A., Stanković, K., Vujisić, M. \& Osmokrović, P. (2011). Novel trends in improvement of solar cell characteristics, Radiation Effects and Defects in Solids, Vol. 166, No. 1, pp. 8-14, (2011), ISSN 1042-0150

Lončar, B., Stanković, S., Vasić, A., \& Osmokrović, P. (2005). The influence of gamma and Xradiation on pre-breakdown currents and resistance of commercial gas filled surge arresters, Nuclear Technology \& Radiation Protection, Vol. XX, No. 1, pp. 59-63, (2005), ISSN 1451-3994

Lončar, B., Osmokrović, P., Vasić, A., \& Stanković, S. (2006). Influence of gamma and X radiation on gas-filled surge arrester characteristics, IEEE Transactions on Plasma Science, Vol. 34, No. 4, pp. 1561-1565, (2006), ISSN 0093-3813

Lončar, B., Osmokrović, P., Vujisić, M., \& Vasić, A. (2007). Temperature and radiation hardness of polycarbonate capacitors, Journal of Optoelectronics and Advanced Materials, Vol. 9, No. 9, pp. 2863-2867, (2007), ISSN 1070-9789

Stojanović, M., Vasić, A., \& Jeynes, C. (1996a). Ion implanted silicides studies by frequency noise level measurements, Nuclear Instruments and Methods B, Vol. 112, pp. 192-195, (1996),ISSN 0168-583X

Stojanović, M., Jeynes, C., Bibić, N., Milosavljević, M., Vasić, A., \& Milošević, Z. (1996b). Frequency noise level of As ion implanted TiN-Ti-Si structures, Nuclear Instruments and Methods B, Vol. 115, pp. 554-556, (1996), ISSN 0168-583X

Stojanović, M., Stanković, S., Vukić, D., Osmokrović, P., Vasić, P., \& Vasić, A. (1998). PV solar systems and development of semiconductor materials, Materials Science Forum, Vols. 282-283, pp. 157-164, (1998), ISSN 0255-5476

Vasić, A., Stojanović, M., Osmokrović, P., \& Stojanović, N. (2000). The influence of ideality factor on fill factor and efficiency of solar cells, Materials Science Forum, Vol. 352, pp. 241-246, (2000), ISSN 0255-5476

Vasić, A., Stanković, S., \& Lončar, B. (2003). Influence of the radiation effects on electrical characteristics of photodetectors, Materials Science Forum, Vol. 413, pp. 171-174, (2004), ISSN 0255-5476

Vasić, A., Osmokrović, P., Stanković, S. \& Lončar, B. (2004). Study of increased temperature influence on the degradation of photodetectors through ideality factor, Materials Science Forum, Vol. 453-454, pp. 37-42, (2004), ISSN 0255-5476

Vasić, A., Osmokrović, P., Lončar, B., \& Stanković, S. (2005). Extraction of parameters from I$\mathrm{V}$ data for nonideal photodetectors: a comparative study, Materials Science Forum, Vol. 494, pp. 83-88, (2005), ISSN 0255-5476

Vasić, A., Vujisić, M., Lončar, B., \& Osmokrović, P. (2007). Aging of solar cells under working conditions, Journal of Optoelectronics and Advanced Materials, Vol. 9 , No. 6, pp. 1843-1846, (2007), ISSN 1070-9789

Vasić, A., Osmokrović, P., Vujisić, M., Dolićanin, C., \& Stanković, K. (2008). Possibilities of improvement of silicon solar cell characteristics by lowering noise, Journal of Optoelectronics and advanced Materials, Vol. 10, No 10, pp. 2800-2804, (2008), ISSN 1070-9789 
Vasic,A., Loncar, B., Vujisic, M., Stankovic, K., \& Osmokrovic, P. (2010). Aging of the Photovoltaic Solar Cells, Proceedings of 27th IEEE International Conference on Microelectronics, pp. 487-490, ISBN 1-4244-0116-x, Nis, Serbia, May 2010 


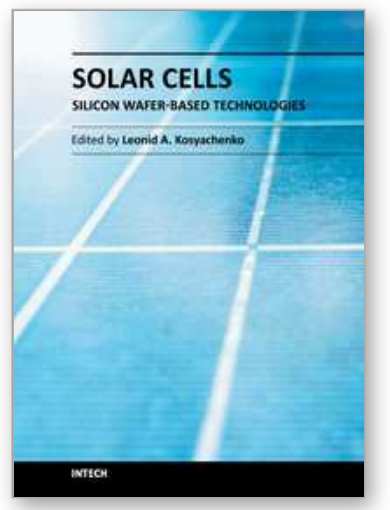

\author{
Solar Cells - Silicon Wafer-Based Technologies \\ Edited by Prof. Leonid A. Kosyachenko
}

ISBN 978-953-307-747-5

Hard cover, 364 pages

Publisher InTech

Published online 02, November, 2011

Published in print edition November, 2011

The third book of four-volume edition of 'Solar Cells' is devoted to solar cells based on silicon wafers, i.e., the main material used in today's photovoltaics. The volume includes the chapters that present new results of research aimed to improve efficiency, to reduce consumption of materials and to lower cost of wafer-based silicon solar cells as well as new methods of research and testing of the devices. Light trapping design in c-Si and $\mathrm{mc}$-Si solar cells, solar-energy conversion as a function of the geometric-concentration factor, design criteria for spacecraft solar arrays are considered in several chapters. A system for the micrometric characterization of solar cells, for identifying the electrical parameters of PV solar generators, a new model for extracting the physical parameters of solar cells, LBIC method for characterization of solar cells, non-idealities in the I-V characteristic of the PV generators are discussed in other chapters of the volume.

\title{
How to reference
}

In order to correctly reference this scholarly work, feel free to copy and paste the following:

Aleksandra Vasic, Milos Vujisic, Koviljka Stankovic and Predrag Osmokrovic (2011). Characterization of Thin Films for Solar Cells and Photodetectors and Possibilities for Improvement of Solar Cells Characteristics, Solar Cells - Silicon Wafer-Based Technologies, Prof. Leonid A. Kosyachenko (Ed.), ISBN: 978-953-307-747-5, InTech, Available from: http://www.intechopen.com/books/solar-cells-silicon-wafer-basedtechnologies/characterization-of-thin-films-for-solar-cells-and-photodetectors-and-possibilities-forimprovement-

\section{INTECH}

open science | open minds

\section{InTech Europe}

University Campus STeP Ri

Slavka Krautzeka 83/A

51000 Rijeka, Croatia

Phone: +385 (51) 770447

Fax: +385 (51) 686166

www.intechopen.com

\section{InTech China}

Unit 405, Office Block, Hotel Equatorial Shanghai

No.65, Yan An Road (West), Shanghai, 200040, China

中国上海市延安西路65号上海国际贵都大饭店办公楼405单元

Phone: +86-21-62489820

Fax: $+86-21-62489821$ 
(C) 2011 The Author(s). Licensee IntechOpen. This is an open access article distributed under the terms of the Creative Commons Attribution 3.0 License, which permits unrestricted use, distribution, and reproduction in any medium, provided the original work is properly cited. 